\title{
INVARIANT BIPARTITE RANDOM GRAPHS ON $\mathbb{R}^{d}$
}

\author{
FABIO LOPES,* Stockholm University
}

\begin{abstract}
Suppose that red and blue points occur in $\mathbb{R}^{d}$ according to two simple point processes with finite intensities $\lambda_{\mathcal{R}}$ and $\lambda_{\mathcal{B}}$, respectively. Furthermore, let $v$ and $\mu$ be two probability distributions on the strictly positive integers with means $\bar{v}$ and $\bar{\mu}$, respectively. Assign independently a random number of stubs (half-edges) to each red (blue) point with law $v$ $(\mu)$. We are interested in translation-invariant schemes for matching stubs between points of different colors in order to obtain random bipartite graphs in which each point has a prescribed degree distribution with law $v$ or $\mu$ depending on its color. For a large class of point processes, we show that such translation-invariant schemes matching almost surely all stubs are possible if and only if $\lambda_{\mathcal{R}} \bar{v}=\lambda_{\mathscr{B}} \bar{\mu}$, including the case when $\bar{v}=\bar{\mu}=\infty$ so that both sides are infinite. Furthermore, we study a particular scheme based on the Gale-Shapley stable marriage problem. For this scheme, we give sufficient conditions on $\nu$ and $\mu$ for the presence and absence of infinite components. These results are two-color versions of those obtained by Deijfen, Holroyd and Häggström.
\end{abstract}

Keywords: Poisson process; random graph; bipartite; stable matching; percolation

2010 Mathematics Subject Classification: Primary 60D05

Secondary 60G55; 05C80

\section{Introduction}

Several random graph models have been applied to the study of complex networks that are observed in nature and human society; for a review, see [8]. Among the models that have found many applications are those which can generate random graphs with a fixed degree distribution. In many applications, e.g. communication networks, a natural development of such models would be to include spatial considerations. A treatable way of doing this is by modeling the vertices of the random graph as the pointset of a point process. Such an approach has the advantage that a well-developed theory of stochastic geometry becomes readily available.

In this work we consider a framework for spatial random graphs with fixed degree distribution that has previously been studied in [2], [6], and [7]. This model has the pointset of a stationary point process in $\mathbb{R}^{d}$ as the vertex set and the edge set is required to be translation invariant. The main contribution of this work is to extend the model to the bipartite context. The bipartiteness appears as the introduction of two types of nodes that may differ in their intensities and probability distributions for their degrees, and the rule that edges between nodes of the same type are not allowed. This extension is a natural setting for many applications, e.g. antennas/receptors (telecommunications), and may generate graphs with different features when compared to the model with a single type of node. Among our contributions are sharp conditions under which the bipartite model is well defined and the study of some percolation questions related to it. In particular, we give considerable attention to a generalization of the so-called two-color stable

Received 5 October 2012; revision received 24 September 2013.

* Postal address: Department of Mathematics, Stockholm University, 10691 Stockholm, Sweden.

Email address: flopes@math.su.se 
matching introduced in [14]. This model is inspired by the Gale-Shapley stable marriage problem [10] which has a long history of applications.

Invariant spatial random graphs have also been studied in other geographies, for example, automorphism invariant random graphs on lattices with fixed degree distribution are studied in [3], [5], and [15], and translation-invariant trees and matchings on point processes in $\mathbb{R}^{d}$ are studied in [9], [12], and [14].

To describe the framework in a bit more detail, let $\mathcal{R}$ and $\mathscr{B}$ (red and blue points) be two translation-invariant simple point processes on $\mathbb{R}^{d}$, jointly ergodic under translations, with finite intensities $\lambda_{\mathcal{R}}$ and $\lambda_{\mathcal{B}}$, respectively. Furthermore, let $v$ and $\mu$ be two probability laws on the strictly positive integers with means $\bar{v}$ and $\bar{\mu}$, respectively. We assign independently to each red (blue) point a random number of stubs (half-edges) with law $v(\mu)$. Our aim is to study schemes for pairing the stubs in order to obtain translation-invariant simple bipartite random graphs whose vertices are the points of $\mathcal{R}$ and $\mathscr{B}$, where the degree of each vertex has law $v$ or $\mu$ depending on its color, and where edges between pairs of points of the same color are not allowed. The first natural question is, how different can the two point processes and their stub laws be for schemes matching almost surely (a.s.) all stubs to be possible? This question was first asked in [2]. Unsurprisingly, a condition relating the number of stubs per point and the intensities of the two point processes must hold in order to match a.s. all stubs. In this work we give a sharp condition for when a matching of a.s. all stubs in the two-color case exists and we extend some of the main results on percolation obtained in [6] for the model with a single Poisson process with fixed degree distribution. We show by examples that, for any given distributions $v$ and $\mu$, there exists a matching scheme that a.s. yields an infinite component as well as only finite components. The properties of the graph hence depend heavily on the matching scheme. Furthermore, for the particular matching scheme based on the Gale-Shapley stable marriage problem [10] mentioned before, we give sufficient conditions for the presence or absence of infinite components.

\subsection{Model and notation}

We now describe our problem and the random objects we will work with more formally. The pointset (or support) of $\mathcal{R}$ is the random set $[\mathcal{R}]:=\left\{x \in \mathbb{R}^{d}: \mathcal{R}(\{x\})>0\right\}$, its points are called red points. Analogously, we write $[\mathscr{B}]$ for the pointset of the process $\mathcal{B}$, and its points are called blue points. In general, for any random point measure $\Lambda$, we write $[\Lambda]$ for its support. The intensity of a translation-invariant point process is the expected number of points in a set of unit volume.

Let $X$ and $Y$ be random variables with law $v$ and $\mu$, respectively, and let $\eta_{\mathcal{R}}$ be a random integer-valued measure on $\mathbb{R}^{d}$ with the same support as $\mathcal{R}$, which, conditionally on $\mathcal{R}$, assigns independent and identically distributed (i.i.d.) values with law $v$ to the elements of $[\mathcal{R}]$. Similarly, for $\mathscr{B}$, let $\eta_{\mathscr{B}}$ assign i.i.d. values with law $\mu$ to the elements of $[\mathcal{B}]$. The pairs $\left(\mathcal{R}, \eta_{\mathcal{R}}\right)$ and $\left(\mathscr{B}, \eta_{\mathscr{B}}\right)$ are two marked point processes with positive integer-valued marks. For $x \in[\mathcal{R}]$, we write $X_{x}$ for $\eta_{\mathcal{R}}(\{x\})$ and, for $y \in[\mathcal{B}]$, we write $Y_{y}$ for $\eta_{\mathcal{B}}(\{y\})$, which we interpret as the number of stubs at the red point $x$ and the number of stubs at the blue point $y$, respectively. Sometimes we refer to the stubs as red or blue depending on the color of the point to which they are assigned. For a marked point process, we refer to the expected number of stubs in a set of unit volume as the stub intensity.

A two-color partial multi-matching scheme for two marked processes $\left(\mathcal{R}, \eta_{\mathcal{R}}\right)$ and $\left(\mathscr{B}, \eta_{\mathcal{B}}\right)$ is a simple point process $\mathcal{M}$ on the space of unordered pairs of points in $\mathbb{R}^{d}$ with the property that, a.s. for every unordered pair $(x, y) \in[\mathcal{M}]$, we have $x \in[\mathcal{R}]$ and $y \in[\mathscr{B}]$, and such 
that in the graph $B$ with vertex set $[\mathcal{R}] \cup[\mathscr{B}]$ and edge set $[\mathcal{M}]$, each vertex $x \in[\mathcal{R}]$ and each vertex $y \in[\mathscr{B}]$ has degree at most $X_{x}$ and $Y_{y}$, respectively. Note that, by definition, the bipartite graph $B$ is simple, that is, it has no self-loops or multiple edges. A two-color partial multi-matching scheme in which a.s. each vertex $x \in[\mathcal{R}]$ and each vertex $y \in[\mathscr{B}]$ has degree $X_{x}$ and $Y_{y}$, respectively, is called a two-color multi-matching scheme. The two-color perfect matching is the special case in which all points of both processes have degree 1 a.s.; this case is studied in detail in [14]. We only consider two-color multi-matching and partial multi-matching schemes that are translation invariant, meaning that $\mathcal{M}$ is invariant in law under the action of all translations of $\mathbb{R}^{d}$. Let $\mathbb{P}$ be the probability measure governing $\left(\mathscr{B}, \eta_{\mathcal{R}}, \mathcal{B}, \eta_{\mathcal{B}}, \mathcal{M}\right)$. We say that a two-color partial multi-matching is a factor if $\mathcal{M}$ is a deterministic function of $\left(\mathscr{B}, \eta_{\mathcal{R}}, \mathcal{B}, \eta_{\mathscr{B}}\right)$, that is, if it does not involve any extra randomness. We introduce the Palm process $\left(\mathscr{B}^{*}, \eta_{\mathcal{R}}^{*}, \mathscr{B}^{*}, \eta_{\mathcal{B}}^{*}, \mathcal{M}^{*}\right)$, with law $\mathbb{P}^{*}$, in which we condition on the presence of a red point at the origin, while taking the mark processes, the pairing scheme, and $\mathscr{B}$ as a stationary background. See, e.g. [1] and [16] for details about Palm processes and point processes. For the Palm version of our process, we denote by $C$ the volume of the component of the red point at the origin, that is, the number of vertices that can be reached by a path in $B\left(\mathcal{R}^{*}, \mathcal{B}^{*}, \mathcal{M}^{*}\right)$ starting at the origin.

Similarly, a one-color (partial) multi-matching of $\left(\mathcal{R}, \eta_{\mathcal{R}}\right)$ is a point process $\mathcal{M}$ on the space of unordered pairs of points in $\mathbb{R}^{d}$, with the property that, a.s. for every pair $(x, y) \in \mathcal{M}$, we have $x, y \in \mathcal{R}$, and such that in the graph $G=G(\mathcal{R}, \mathcal{M})$ with vertex set [R] and edge set $[\mathcal{M}]$, each vertex $x$ has degree (at most) $X_{x}$.

Let $|\cdot|$ denote Euclidean distance. A set $S \subset \mathbb{R}^{d}$ is said to be nonequidistant if there are no distinct points $x, y, u, w \in S$ with $|x-y|=|x-z|$ or $|x-y|=|u-w|$. A descending chain is an infinite sequence $\left\{x_{i}\right\}_{i \geq 1} \subset S$ such that $\left|x_{i}-x_{i-1}\right|$ is strictly decreasing. Most of our results require that the underlying point processes have support that is a.s. nonequidistant and that has no descending chains. We observe that both these conditions hold for Poisson processes; for a proof, see [11].

\subsection{Results}

Our first result gives a necessary and sufficient condition for the existence of a two-color multi-matching scheme for two marked point processes $\left(\mathcal{R}, \eta_{\mathcal{R}}\right)$ and $\left(\mathscr{B}, \eta_{\mathscr{B}}\right)$.

Theorem 1.1. Let $\left(\mathcal{R}, \eta_{\mathcal{R}}\right)$ and $\left(\mathcal{B}, \eta_{\mathscr{B}}\right)$ be two marked point processes on $\mathbb{R}^{d}$, jointly ergodic under translations, with simple ground processes of finite intensities $\lambda_{\mathcal{R}}$ and $\lambda_{\mathcal{B}}$, and with i.i.d., positive, integer-valued marks with laws $v$ and $\mu$, respectively. Suppose that, a.s., $[\mathcal{R}] \cup[\mathcal{B}]$ is nonequidistant and has no descending chains. Then there exists a two-color multi-matching scheme for $\left(\mathcal{R}, \eta_{\mathcal{R}}\right)$ and $\left(\mathcal{B}, \eta_{\mathcal{B}}\right)$ if and only if

$$
\lambda_{\mathcal{R}} \bar{v}=\lambda_{\mathcal{B}} \bar{\mu} .
$$

In (1.1) we also allow $\infty$ on both sides, which occurs when both processes have marks with infinite mean.

The only-if part follows from a simple application of the so-called mass transport principle (see Lemma 2.1 below). For the if part, we present an algorithm leading to a two-color stable multi-matching, defined as follows (See Section 2),

Definition 1.1. A two-color (partial) multi-matching scheme $\mathcal{M}$ is said to be stable if, a.s. for any pair of points $x \in[\mathcal{R}]$ and $y \in[\mathcal{B}]$, either they are linked by an edge or at least one of them has no incident edges longer than $|x-y|$. 
Let $\mathcal{M}$ be a two-color stable (partial) multi-matching, and denote by $\mathcal{M}_{1}(x)$ the most distant partner of $x \in[\mathcal{R}] \cup[\mathscr{B}]$ in $\mathcal{M}$. If $x$ has unmatched stubs in $\mathcal{M}, \mathcal{M}_{1}(x)=\infty$. We say that $x \in[\mathcal{R}] \cup[\mathcal{B}]$ in $\mathcal{M}$ desires a point $y \in \mathbb{R}^{d}$ if $\left|x-\mathcal{M}_{1}(x)\right|>|x-y|$. Note that the definition of stability is equivalent to the assertion that any two points of different colors in $[\mathcal{R}] \cup[\mathcal{B}]$ that desire each other are matched in $\mathcal{M}$.

When the degree distributions have finite means, the proof of the fact that the algorithm presented in Section 2 yields a perfect matching is an extension of the arguments in [14]. When the degree distributions have infinite mean, however, a different approach is required and our argument here is inspired by work on continuum percolation.

Next, we state our results on the percolation properties of two-color multi-matchings. For that, we leave the setting of more general point processes and work with two independent marked Poisson processes. As mentioned before, the results extend analogous results proved in [6] for the one-color case. The first result illustrates the fact that the percolation properties of the model depend heavily on the matching scheme.

Theorem 1.2. Let $\left(\mathcal{R}, \eta_{\mathcal{R}}\right)$ and $\left(\mathcal{B}, \eta_{\mathcal{B}}\right)$ be two independent marked Poisson processes on $\mathbb{R}^{d}, d \geq 1$, with finite intensities $\lambda_{\mathcal{R}}$ and $\lambda_{\mathscr{B}}$, respectively, satisfying (1.1).

(a) If $\left(\mathcal{R}, \eta_{\mathcal{R}}\right)$ and $\left(\mathscr{B}, \eta_{\mathcal{B}}\right)$ have the same law, then there exists a simple translation-invariant factor matching scheme such that $\mathbb{P}^{*}(C<\infty)=1$.

(b) If $\mathbb{P}(Y \geq 2) \geq \mathbb{P}(X \geq 2)>0$ then there exists a simple translation-invariant factor matching scheme with $\mathbb{P}^{*}\left(C=\infty \mid X_{0} \geq 2\right)=1$.

The next result gives sufficient conditions on the degree distributions that guarantee the existence and nonexistence of a component with infinitely many vertices for two-color stable multi-matchings in the Poisson case.

Theorem 1.3. Let $\left(\mathcal{R}, \eta_{\mathcal{R}}\right)$ and $\left(\mathcal{B}, \eta_{\mathcal{B}}\right)$ be two marked Poisson processes on $\mathbb{R}^{d}$, jointly ergodic under translations, with finite intensities $\lambda_{\mathcal{R}}$ and $\lambda_{\mathcal{B}}$, and i.i.d. marks with laws $v$ and $\mu$ both with finite mean, satisfying (1.1). Consider two-color stable multi-matchings.

(a) For any $d \geq 2$, there exists a $k=k(d)$ such that if $\mathbb{P}(Y \geq k)=\mathbb{P}(X \geq k)=1$ then $\mathbb{P}^{*}(C=\infty)>0$.

(b) For any $d \geq 1$, if $\mathbb{P}(Y \leq 2)=\mathbb{P}(X \leq 2)=1$ and $\mathbb{P}(Y=1)>0$, then $\mathbb{P}^{*}(C=\infty)=0$.

Although these results are similar to the one-color versions in [6], we note that there are differences between the one-color model and the two-color model. Indeed, for the case with constant degree 2 on $\mathbb{R}$, Deijfen et al. [7] provided compelling evidence that the one-color model generates an infinite component, while it was proved in [4] that the two-color model does not.

The rest of the paper is organized as follows. In Section 2 we present the algorithm leading to a two-color stable multi-matching and prove Theorem 1.1. In Section 3 we prove Theorems 1.2 and 1.3. Finally, in Section 4 we pose some questions.

\section{Two-color stable multi-matching}

We now describe an iterative procedure that generates a two-color stable multi-matching for the two marked point processes $\left(\mathcal{R}, \eta_{\mathcal{R}}\right)$ and $\left(\mathscr{B}, \eta_{\mathcal{B}}\right)$. We refer to this procedure as the two-color iterated mutually closest multi-matching algorithm (2CIMC). The procedure is a twocolor extension of the algorithm proposed in [2] for one-color multi-matchings. That algorithm, 
in turn, is a multi-matching generalization of the iterated mutually closest matching algorithm from [14].

Following [14], we call a pair of points $x$ and $y$ potential partners if one is red while the other is blue. We also say that two potential partners $x$ and $y$ are mutually closest if $y$ is the closest potential partner to $x$ and $x$ is the closest potential partner to $y$.

Given the point configurations $[\mathcal{R}]$ and $[\mathcal{B}]$, in the first step of the algorithm we create an edge between each pair of mutually closest potential partners in $[\mathcal{R}] \cup[\mathcal{B}]$ and remove one stub from each of these points. Then in the $i$ th step we consider the set of points which still have at least one stub after the previous steps. We call two potential partners compatible if no edge has been created between them in the previous steps. We create an edge between each pair of compatible mutually closest potential partners in this set, and again we remove one stub from each of these points. This is then iterated indefinitely. The limiting resulting graph $B$ obtained after an infinite number of iterations is the graph with vertex set $[\mathcal{R}] \cup[\mathscr{B}]$ and edge set equal to the union of all edges created in each step.

By construction, the algorithm above a.s. yields a two-color partial multi-matching that avoids multiple edges and self-loops. The next result shows that if the two processes have the same finite stub intensity then a.s. all stubs are matched and we obtain a two-color stable multi-matching. In the case where the stub intensities are different we a.s. obtain a two-color partial stable multi-matching that exhausts all stubs in the process with smaller stub intensity.

Proposition 2.1. Let $\left(\mathcal{R}, \eta_{\mathcal{R}}\right)$ and $\left(\mathcal{B}, \eta_{\mathscr{B}}\right)$ be two marked point processes on $\mathbb{R}^{d}$, jointly ergodic under translations, with simple ground processes with finite intensities $\lambda_{\mathcal{R}}$ and $\lambda_{\mathscr{B}}$, and i.i.d marks with laws $v$ and $\mu$, respectively. Suppose that, a.s., $[\mathcal{R}] \cup[\mathscr{B}]$ is nonequidistant and has no descending chains.

(a) If (1.1) holds then, a.s., the 2CIMC algorithm described above exhausts the set of stubs, and the limiting graph (after an infinite number of iterations) is a two-color stable multimatching. No other two-color stable multi-matching of $\left(\mathcal{R}, \eta_{\mathcal{R}}\right)$ and $\left(\mathscr{B}, \eta_{\mathscr{B}}\right)$ exists.

(b) If (1.1) does not hold then the 2CIMC algorithm yields a translation-invariant two-color partial stable multi-matching scheme that a.s. exhausts all stubs in the process with smaller stub intensity.

Remark 2.1. For the case when $v(\{1\})=\mu(\{1\})=1$, the result is an application of the two-color stable matching of [14, Proposition 9].

Remark 2.2. We note that the 2 CIMC algorithm can also be applied when some pairs of vertices already have an edge between them and additional connections between such vertices are prohibited. When the existing edges form a translation-invariant process, the same argument as used in the proof of Proposition 2.1 shows that the procedure yields a two-color partial multimatching that a.s. exhausts all stubs of the process with lower stub intensity.

We state without proof the following well-known lemma, referred to as the mass transport principle; see, e.g. [14, Lemma 8(ii)]. Let $Q$ denote the unit cube $[0,1)^{d}$.

Lemma 2.1. (Mass transport principle.) For $d \geq 1$, suppose that $T$ is a random nonnegative measure on $\mathbb{R}^{d} \times \mathbb{R}^{d}$ such that $T(A, B):=T(A \times B)$ and $T(A+w, B+w)$ are equal in law for all $w \in \mathbb{Z}^{d}$. Then

$$
\mathbb{E} T\left(Q, \mathbb{R}^{d}\right)=\mathbb{E} T\left(\mathbb{R}^{d}, Q\right)
$$


For Borel sets $A, B \subset \mathbb{R}^{d}$, it may be helpful to interpret $T(A, B)$ as the amount of mass transported from $A$ to $B$. The following lemma is a multi-matching analogue of [14, Proposition 7], and gives an immediate corollary, which establishes the only-if part of Theorem 1.1.

Lemma 2.2. (Fairness lemma.) Let $\left(\mathcal{R}, \eta_{\mathcal{R}}\right)$ and $\left(\mathcal{B}, \eta_{\mathscr{B}}\right)$ be two marked point processes on $\mathbb{R}^{d}$ with simple ground processes with finite intensities $\lambda_{\mathcal{R}}$ and $\lambda_{\mathscr{B}}$, and i.i.d. marks with laws $v$ and $\mu$, respectively. Let $\mathcal{M}$ be a translation-invariant two-color partial multi-matching of the two marked processes. Then the expected number of matched stubs incident to red points in $Q$ is equal to the expected number of matched stubs incident to blue points in $Q$.

Proof. Apply Lemma 2.1 to the mass transport in which each red point in A with matched stubs sends unit mass to each of its partners in B.

Corollary 2.1. If there exists a translation-invariant two-color multi-matching for $\left(\mathcal{R}, \eta_{\mathcal{R}}\right)$ and $\left(\mathscr{B}, \eta_{\mathcal{B}}\right)$, then $\lambda_{\mathcal{R}} \mathbb{E}(X)$ and $\lambda_{\mathcal{B}} \mathbb{E}(Y)$ must be equal.

Proof of Proposition 2.1(a) when both sides of (1.1) are finite. Note that nonequidistance ensures that the algorithm is a.s. well defined. Let $\mathcal{N}_{\mathcal{R}}$ and $\mathcal{N}_{\mathcal{B}}$ be the point processes of the red and blue points, respectively, with at least one unmatched stub after the 2CIMC algorithm is completed. Then $\mathcal{N}_{\mathcal{R}}$ and $\mathcal{N}_{\mathcal{B}}$ are ergodic point processes, and, hence, we have three possible cases: (i) both have a.s. infinitely many points, (ii) one has a.s. infinitely many points and the other has a.s. no points, (iii) both have a.s. no points. Our aim is to rule out the first two cases: (i) the first case is ruled out by a simple adaptation of [6, Proposition 2.2]; (ii) the second case is ruled out by adapting the argument in the proof of [14, Proposition 9] which shows a similar result for two-color stable matchings. If we assume that both processes $\left(\mathcal{R}, \eta_{\mathcal{R}}\right)$ and $\left(\mathscr{B}, \eta_{\mathcal{B}}\right)$ satisfy (1.1) then Lemma 2.2 implies that after the 2 CIMC algorithm the expected number of remaining red stubs and blue stubs in $Q$ must be equal. Since this is not the case if one of them has a.s. infinitely many points with unmatched stubs and the other is a.s. empty, this case is ruled out. Hence, we can conclude that both $\left[\mathcal{N}_{\mathcal{R}}\right]$ and $\left[\mathcal{N}_{\mathscr{B}}\right]$ have a.s. no points.

That the resulting two-color multi-matching is stable follows from the definition of stability. Furthermore, as in [14, Lemma 15], it follows from induction over the steps in the algorithm that each edge in the resulting graph must be present in every two-color stable multi-matching for this given configuration of points and stubs. The last claim follows from the inductive hypothesis and stability, and implies that the two-color stable multi-matching is a.s. unique.

Remark 2.3. We remark that in general stable marriage problems do not have unique solutions; see [10]. The key to uniqueness in our setting is that the preferences are based on distance, and that the pointset is nonequidistant and does not contain descending chains; see [14, Discussion following Lemma 15]. It is not difficult to give examples of pointsets containing descending chains or where the nonequidistance does not hold for which uniqueness or existence of a two-color stable multi-matching fails.

When both sides of (1.1) are infinite, we cannot use the same argument as in the finite case to rule out (ii). Instead, we use an argument inspired by the proof of the following result for the Boolean model. Let $\mathcal{X}$ be an ergodic point process in $\mathbb{R}^{d}$ to which we assign balls centered at the points of $[X]$ with i.i.d. radius distributed as a random variable $\rho$. If $\mathbb{E}\left(\rho^{d}\right)=\infty$ then $\mathbb{P}\left(\bigcup_{x \in[\mathcal{X}]} B\left(x, \rho_{x}\right)=\mathbb{R}^{d}\right)=1$; see [17, Proposition 3.1].

Proof of Proposition 2.1(a) when both sides of (1.1) are infinite. We define $\mathcal{N}_{\mathcal{R}}$ and $\mathcal{N}_{\mathscr{B}}$ as before. We will prove that if $\mathbb{E}(X)=\mathbb{E}(Y)=\infty$ then any point in $\mathbb{R}^{d}$ is desired by infinitely many red points and infinitely many blue points a.s. This implies that $\mathcal{N}_{\mathcal{R}}$ and $\mathcal{N}_{\mathcal{B}}$ must be 
empty a.s., since otherwise, each point in $\mathcal{N}_{\mathcal{R}}$ and $\mathcal{N}_{\mathcal{B}}$ would be matched to infinitely many points of opposite color, contradicting the fact that the initial number of stubs per point is a.s. finite. Recall that $\mathcal{M}_{1}(x)$ denotes the most distant $\mathcal{M}$-partner of $x \in[\mathcal{R}] \cup[\mathcal{B}]$ in $\mathcal{M}$.

For each $x \in[\mathcal{R}]$, let $r_{x}$ be the radius of the smallest ball centered at $x$ containing $\eta_{\mathcal{R}}(x)$ blue points. It is clear that $r_{x} \leq\left|x-\mathcal{M}_{1}(x)\right|$ for all $x \in[\mathcal{R}]$. Hence,

$$
\bigcup_{x \in[\mathcal{R}]} B\left(x, r_{x}\right) \subset \bigcup_{x \in[\mathcal{R}]} B\left(x,\left|x-\mathcal{M}_{1}(x)\right|\right) .
$$

We show that $\mathbb{E}(X)=\infty$ implies that, a.s., any bounded region is covered by infinitely many balls $B\left(x, r_{x}\right)$ with $x \in[\mathcal{R}]$; we refer to these as red balls. The proof for blue balls is analogous.

We consider a sequence of nonrandom balls $\left\{C_{n}\right\}_{n \geq 0}$ centered at the origin and with radius $2^{n / d}$. For any Borel set $A$, let $\mathcal{L}(A)$ be its Lebesgue measure. From the ergodic theorem, we have, for large enough $n$ (depending on the realisation),

$$
\lambda_{\mathcal{R}} \frac{3}{4} V_{n} \leq \mathcal{R}\left(C_{n}\right) \leq \lambda_{\mathcal{R}} \frac{5}{4} V_{n} \quad \text { and } \quad \lambda_{\mathcal{B}} \frac{3}{4} V_{n} \leq \mathcal{B}\left(C_{n}\right) \leq \lambda_{\mathcal{B}} \frac{5}{4} V_{n},
$$

where $V_{n}=\mathcal{L}\left(C_{n}\right)$. Note that $\mathcal{L}\left(C_{n+1}\right)=c_{d} 2^{n+1}=2 \mathcal{L}\left(C_{n}\right)$, where $c_{d}$ is a constant depending only on $d$.

Now, for large enough $n$, the spherical shell $C_{n} \backslash C_{n-1}$ contains at least $\frac{1}{4} \lambda_{\mathcal{R}} V_{n}$ red points, and this bound can be written as $b\left(d, \lambda_{\mathcal{R}}\right) 2^{n}$, where $b\left(d, \lambda_{\mathcal{R}}\right)$ is a constant depending only on $d$ and $\lambda_{\mathcal{R}}$. Let $\left\{W_{n}\right\}_{n \geq 0}$ be the sequence of nonrandom balls $B\left(0,\left(2^{n / d}+1\right) 2\right)$. Again, for large enough $n$, we have $\mathscr{B}\left(W_{n}\right) \leq w\left(d, \lambda_{\mathcal{B}}\right) 2^{n}$ for another constant $w\left(d, \lambda_{\mathscr{B}}\right)$ depending only on $d$ and $\lambda_{\mathscr{B}}$. Note that each ball $W_{n}$ contains all possible balls centered at a point of $C_{n} \backslash C_{n-1}$ which do not cover $C_{0}$ completely.

Let $H$ be the event that $C_{0}$ is completely covered by red balls only finitely many times. Furthermore, let $E_{n}$ be the event that $C_{0}$ is not completely covered by a ball which is centered in $C_{n} \backslash C_{n-1}$, and let $A_{m}$ be the event that $m$ is the first index such that

$$
\mathcal{R}\left(C_{n} \backslash C_{n-1}\right) \geq b\left(d, \lambda_{\mathcal{R}}\right) 2^{n} \text { and } \mathcal{B}\left(W_{n}\right) \leq w\left(d, \lambda_{\mathcal{B}}\right) 2^{n}
$$

for all $n \geq m$. The $A_{m}$ events form a partition of the probability space. We write

$$
\mathbb{P}\left(H \mid A_{m}\right) \leq \sum_{L=0}^{\infty} \mathbb{P}\left(\bigcap_{k=L+1}^{\infty} E_{k} \mid A_{m}\right) \leq \sum_{L=0}^{\infty} \mathbb{P}\left(\bigcap_{k=(L+1) \vee m}^{\infty} E_{k} \mid A_{m}\right) .
$$

Below we show that each of the terms in the above sum is equal to 0 . First, we observe that, given $A_{m}$ and for large enough $n$, the event $E_{n}$ implies that, for each red point $y \in C_{n} \backslash C_{n-1}$, the ball $B\left(y, r_{y}\right)$ is contained in $W_{n}$. Hence, each such point $y$ has at most $w\left(d, \lambda_{\mathcal{B}}\right) 2^{n}$ stubs attached. For any fixed $L$ and $m$, write $M=(L+1) \vee m$. Then we can bound

$$
\begin{aligned}
\mathbb{P}\left(\bigcap_{k=M}^{\infty} E_{k} \mid A_{m}\right) \\
\quad \leq \mathbb{P}\left(\bigcap_{k=M}^{\infty}\left\{\text { all balls centered at red points of } C_{k} \backslash C_{k-1} \text { are in } W_{k}\right\} \mid A_{m}\right) \\
\left.\quad \leq \mathbb{P}\left(\bigcap_{k=M}^{\infty} \text { each red point of } C_{k} \backslash C_{k-1} \text { has at most }\left\lceil w\left(d, \lambda_{\mathcal{B}}\right)\right\rceil 2^{k} \text { stubs }\right\} \mid A_{m}\right) \\
\quad \leq \prod_{k=M}^{\infty} \mathbb{P}\left(X \leq\left\lceil w\left(d, \lambda_{\mathcal{B}}\right)\right\rceil 2^{k}\right)^{b\left(d, \lambda_{\mathcal{R}}\right) 2^{k}}
\end{aligned}
$$




$$
\begin{aligned}
& =\left\{\prod_{k=M}^{\infty} \mathbb{P}\left(X \leq\left\lceil w\left(d, \lambda_{\mathcal{B}}\right)\right\rceil 2^{k}\right)^{\left\lceil w\left(d, \lambda_{\mathcal{B}}\right)\right\rceil 2^{k}}\right\}^{b\left(d, \lambda_{\mathcal{R}}\right) /\left\lceil w\left(d, \lambda_{\mathcal{B}}\right)\right\rceil} \\
& \leq\left\{\prod_{k=M}^{\infty} \mathbb{P}\left(X \leq\left\lceil w\left(d, \lambda_{\mathcal{B}}\right)\right\rceil 2^{M}\right) \cdots \mathbb{P}\left(X \leq\left\lceil w\left(d, \lambda_{\mathcal{B}}\right)\right\rceil 2^{M+1}-1\right)\right\}^{b\left(d, \lambda_{\mathcal{R}}\right) /\left\lceil w\left(d, \lambda_{\mathcal{B}}\right)\right\rceil} \\
& =\left\{\prod_{k=\left\lceil w\left(d, \lambda_{\mathcal{B}}\right)\right\rceil 2^{M}}^{\infty}(1-\mathbb{P}(X>k))\right\}^{b\left(d, \lambda_{\mathcal{R}}\right) /\left\lceil w\left(d, \lambda_{\mathcal{B}}\right)\right\rceil} \cdot
\end{aligned}
$$

In the third inequality we used the independence of the number of stubs at each red point and the point processes, and the fact that, conditional on $A_{m}$, there are at least $b\left(d, \lambda_{\mathcal{R}}\right) 2^{k}$ red points in $C_{k} \backslash C_{k-1}$ for $k \geq m$. This probability is equal to 0 if and only if $\sum_{k=\left\lceil w\left(d, \lambda_{\mathcal{B}}\right)\right\rceil 2^{M}}^{\infty} \mathbb{P}(X>k)=$ $\infty$, which is equivalent to $\mathbb{E}(X)=\infty$. Hence, any point in $C_{0}$ is covered by infinitely many red balls a.s., and by translation invariance and (2.1), it follows that, a.s., any blue point in $[\mathcal{B}]$ is desired by infinitely many red points. The uniqueness and stability of $\mathcal{M}$ follows as in the case when the expected numbers of stubs per point in both processes are finite.

Proof of Proposition 2.1(b). The proof uses the same arguments as those in the proof of Proposition 2.1(a).

\section{Percolation for the Poisson case}

In this section we prove Theorems 1.2 and 1.3.

\subsection{Percolating and nonpercolating schemes}

Next we describe two factor schemes: one that a.s. yields only finite components, and another which a.s. gives at least one infinite component.

Proof of Theorem 1.2(a). Let $\mathcal{R}_{n}$ and $\mathcal{B}_{n}$ denote the processes of red and blue points with exactly $n$ stubs, respectively. The idea is to partition $\left[\mathcal{R}_{n}\right] \cup\left[\mathcal{B}_{n}\right]$ into groups of size $2 n$ where each group has $n$ points of each color to construct complete bipartite graphs on each of these groups.

Take $n$ such that $\mathcal{R}_{n}$ and $\mathcal{B}_{n}$ are nonempty; this is possible since $\mu=v$. First, to partition $\left[\mathcal{R}_{n}\right]$, we assign each red point in $\left[\mathcal{R}_{n}\right]$ a type $i \in\{1,2, \ldots, n\}$ as follows. Let $D_{\mathcal{R}_{n}}^{*}$ denote the distance from the origin to the closest point in the Palm version of $\mathcal{R}_{n}$, and let $0=d_{0}, d_{1}, \ldots, d_{n-1}, d_{n}=\infty$ be such that

$$
\mathbb{P}^{*}\left(d_{i-1}<D_{\mathcal{R}_{n}}^{*} \leq d_{i}\right)=\frac{1}{n}, \quad i=1, \ldots, n .
$$

For $x \in\left[\mathcal{R}_{n}\right]$, let $D_{\mathcal{R}_{n}}(x)$ denote the distance to the nearest point in $\left[\mathcal{R}_{n}\right]$. We assign $x \in\left[\mathcal{R}_{n}\right]$ type $i$ if $d_{i-1}<D_{\mathcal{R}_{n}}(x) \leq d_{i}$, and let $\mathcal{R}_{n}^{i}$ be the process of points of $\mathcal{R}_{n}$ of type $i$. Since the processes have the same law, we can use the same numbers $\left\{d_{0}, d_{1}, \ldots, d_{n}\right\}$ to partition $\mathcal{B}_{n}$. Analogously, for $y \in\left[\mathscr{B}_{n}\right]$, we define $D_{\mathscr{B}_{n}}(y)$ and assign the type $i$ if $d_{i-1}<D_{\mathcal{B}_{n}}(y) \leq d_{i}$. Note that the assignment of types does not involve any extra randomness, and that, for each $n$, the processes $\mathcal{R}_{n}^{1}, \mathcal{B}_{n}^{1}, \ldots, \mathcal{R}_{n}^{n}, \mathcal{B}_{n}^{n}$ have equal intensities and are jointly ergodic under translations. Since all processes have the same intensity, we can use the two-color stable matching repeatedly to construct groups of size $2 n$ as follows. First, for each color, we use the two-color stable matching to match each type- $i$ point to a unique type- $(i+1)$ point for $i=1,2, \ldots, n-1$. The union of these matchings partitions $\left[\mathcal{R}_{n}\right] \cup\left[\mathcal{B}_{n}\right]$ into monocromatic 
groups of size $n$. To form groups of size $2 n$ with $n$ points of each color, we assign to each red point of type 1 a blue point of type 1, again using the two-color stable matching.

Remark 3.1. The same idea can be applied to cover some asymmetric cases satisfying (1.1); however, we do not have a general construction for the asymmetric case.

In order to prove part (b) of Theorem 1.2, we make use of the following result of [12, Theorem 1].

Theorem 3.1. (Holroyd and Peres.) Let $\mathcal{P}$ be a Poisson process on $\mathbb{R}^{d}$.

(a) $\mathcal{P}$ has a factor graph which is a.s. a locally finite one-ended tree.

(b) $\mathcal{P}$ has a factor graph which is a.s. a directed doubly infinite path.

Part (b) of Theorem 3.1 is obtained from part (a) as follows. Once the one-ended tree is given, a doubly infinite path is obtained by first ordering the children of each vertex according to the distance from its parent, and then ordering all vertices according to a depth-first search. By creating an edge between each pair of vertices that fall next to each other in this ordering we obtain the desired doubly infinite path; see [12] for details. In the proof of Theorem 1.2(b) we construct a doubly infinite path with alternating colors. The proof relies on Theorem 3.1, but we have to make an intermediate step when going from the tree to the doubly infinite path.

Proof of Theorem 1.2(b). First, we will prove the claim for the symmetric case. Let $\mathcal{R}_{\geq 2}$ denote the process of red points $x \in[\mathcal{R}]$ with $X_{x} \geq 2$, and define $\mathscr{B}_{\geq 2}$ similarly. Since both processes have the same intensity, there is a two-color perfect matching $\mathcal{M}$ between them. We use Theorem 3.1(a) to obtain a translation-invariant one-ended tree $\mathcal{T}$ for the Poisson process $\mathscr{B}_{\geq 2}$. To obtain a doubly infinite path, we create an edge between each blue vertex in $\mathcal{T}$ and the $\mathcal{M}$-partner of the next blue vertex of $\mathcal{T}$ in the ordering mentioned above to obtain Theorem 3.1(b). We are left with a doubly infinite path with alternating colors involving all points of $\left[\mathcal{R}_{\geq 2}\right] \cup\left[\mathcal{B}_{\geq 2}\right]$. When this is done, we remove two stubs from each point in $\left[\mathcal{R}_{\geq 2}\right] \cup\left[\mathcal{B}_{\geq 2}\right]$. In order to match the points of $[\mathcal{R}] \cup[\mathcal{B}]$ with remaining stubs, we apply the 2CIMC procedure with the restriction that we do not allow connections between points that already have an edge between them arising from the connections along the doubly infinite path. Since, by Lemma 2.2, the processes of red and blue points with remaining stubs have equal stub intensity, we can use Proposition 2.1 with Remark 2.2 to match a.s. all remaining stubs.

For the asymmetric case, we use the marked point process with the lower intensity of points with degree greater than or equal to 2 to obtain the tree $\mathcal{T}$. The rest of the proof is analogous to the symmetric case.

\subsection{Percolation for the stable multi-matching}

We now show that the sufficient conditions given in [6] for the existence and absence of an infinite component for the one-color stable multi-matching on a Poisson process with i.i.d. degrees can be extended to our model.

Proof of Theorem 1.3(a). The result follows from simple changes in the proof of [6, Theorem 1.2(a)]. The key to the proof is a renormalization procedure partitioning $\mathbb{R}^{d}$ into cubes. Our modification lies in changing the definition of an acceptable cube. Here a cube is acceptable if it contains at least one point of each color but not 'too many' of each color, where 'too many' will be determined by the renormalization procedure. The probability that a cube is acceptable can be made as close to 1 as we like by adjusting the renormalization grid. With this at hand, 
the line of the argument is the same as in [6, Theorem 1.2(a)] and we refer the reader there for details.

Next, we prove Theorem 1.3(b). A modification of the argument in [6, Theorem 1.2(b)] allows us to prove the claim under the assumption that only one of the processes has a strictly positive probability of degree 1 (if both processes have strictly positive probability of degree 1 , it follows from the same argument as in [6]).

The next lemma states that the only infinite components that can appear in the graph $B$ obtained from a translation-invariant multi-matching in which all points have degree at most 2 are a.s. bi-infinite paths. This follows from the mass-transport principle; see [6, Lemma 5.1].

Lemma 3.1. In any translation-invariant two-color multi-matching scheme, $B$ a.s. has no component consisting of a singly infinite path.

Proof of Theorem 1.3(b). Assume for contradiction that bi-infinite paths occur with positive probability. For any configuration with at least one bi-infinite path, let $\left\{x_{i}\right\}_{i=-\infty}^{\infty}$ be the biinfinite path with the nearest vertex to the origin, and write $r$ for the second closest red point to the origin on such a bi-infinite path, and $b_{1}$ and $b_{2}$, respectively, for its two blue neighbors on the path. In a coupled configuration, this path will be cut apart into two singly infinite paths by removing the red point $r$ and by re-randomizing the degree of the blue points. This contradicts Lemma 3.1.

Let $\left\{Y_{y}\right\}_{y \in[\mathscr{B}]}$ be the degree process associated with $\left(\mathscr{B}, \eta_{\mathscr{B}}\right)$. First, construct a coupled configuration in which we introduce for the blue points a modified degree process $\left\{\tilde{Y}_{y}\right\}_{y \in[\mathscr{B}]}$ with the same law as in the original configuration, and remove the red point $r$. For each $y \in[\mathscr{B}]$, we let $\tilde{Y}_{y}=Y_{y}$ with probability $1-\mathrm{e}^{-|y|}$, and with the remaining probability we independently generate a new number of stubs with law $\mu$. The points which have received a newly generated degree are called re-randomized points. Now, the same Borel-Cantelli argument as used in [6, Theorem 1.2(b)] shows that the number of re-randomized points in the modified configuration is a.s. finite. In addition, we can obtain that the law of the coupled configuration is absolutely continuous with respect to the law of $\left(\mathcal{R}, \eta_{\mathcal{R}}, \mathcal{B}, \eta_{\mathcal{B}}\right)$ by using [13, Theorem 1 and Lemma 3.3] in a similar way as in [13, Proof of Lemma 6.2].

Let $A$ be the event that in the coupled configuration the only re-randomized points are $b_{1}$ and $b_{2}$, and that both these points have received a newly generated degree equal to 1 . By the finiteness of the set of re-randomized blue points, this event has positive probability. Now we claim that, under $A$, the two-color stable multi-matching obtained in the coupled configuration is equal to that obtained in the original configuration except for the removed red point $r$ and its incident edges $\left(r, b_{1}\right)$ and $\left(r, b_{2}\right)$ that do not exist. We prove our claim in two steps.

First, write $B$ for the resulting graph obtained from the original configuration and write $\tilde{B}$ for the graph obtained by removing the point $r$ and its incident edges from $B$. From the definition of stability we can see that $\tilde{B}$ is stable since it inherits from $B$ the property that, for any unmatched pair of compatible points, at least one has no edges longer than their distances. Second, from Proposition 2.1, the two-color stable multi-matching is a.s. unique and is attainable by the 2CIMC algorithm. Hence, by applying such an algorithm, under $A$, to the coupled configuration a.s. leads to a graph which must be equal to $\tilde{B}$. This concludes the proof.

\section{Questions}

(i) Let $T$ be the total edge length of a typical red point, that is, the sum of the length of all edges incident to it. In [2], it was shown that there exists a translation-invariant one-color 
multi-matching scheme with $\mathbb{E}^{*}(T)<\infty$ if and only if $\mathbb{E}\left(X^{(d+1) / d}\right)<\infty$. Is there a similar result for two-color multi-matchings?

(ii) Consider the two-color stable multi-matching. For $d=1$, is there any sufficient condition to a.s. obtain an infinite component? Also, for $d \geq 1$, is $\mathbb{E}(X)=\mathbb{E}(Y)=\infty$ a sufficient condition for the existence of an infinite component?

\section{Acknowledgements}

I thank my thesis advisor Mia Deijfen and an anonymous referee for their useful comments. I am also grateful to Alexander Holroyd, who to the best of my knowledge first suggested condition (1.1) of Theorem 1.1.

\section{References}

[1] Daley, D. J. And Vere-Jones, D. (2008). An Introduction to the Theory of Point Processes, Vol. II, General Theory and Structure, 2nd edn. Springer, New York.

[2] Deijfen, M. (2009). Stationary random graphs with prescribed iid degrees on a spatial Poisson process. Eletron. Commun. Prob. 14, 81-89.

[3] Deijfen, M. And Jonasson, J. (2006). Stationary random graphs on $\mathbb{Z}$ with prescribed iid degrees and finite mean connections. Eletron. Commun. Prob. 11, 336-346.

[4] Deijfen, M. And Lopes, F. M. (2012). Bipartite stable Poisson graphs on R. Markov Process. Relat. Fields 18, 583-594.

[5] Deijfen, M. and Meester, R. (2006). Generating stationary random graphs on $\mathbb{Z}$ with prescribed independent, identically distributed degrees. Adv. Appl. Prob. 38, 287-298.

[6] Deijfen, M., Häggström, O. And Holroyd, A. E. (2012). Percolation in invariant Poisson graphs with i.i.d. degrees. Ark. Mat. 50, 41-58.

[7] Deijfen, M., Holroyd, A. E. And Peres, Y. (2011). Stable Poisson graphs in one dimension. Eletron. J. Prob. 16, 1238-1253.

[8] Durrett, R. (2007). Random Graph Dynamics. Cambridge University Press.

[9] Ferrari, P. A., Landim, C. AND Thorisson, H. (2004). Poisson trees, succession lines and coalescing random walks. Ann. Inst. H. Poincaré Prob. Statist. 40, 141-152.

[10] Gale, D. and Shapley, L. S. (1962). College admissions and the stability of marriage. Amer. Math. Monthly 69, 9-15.

[11] HäGgStröm, O. AND MeEster, R. (1996). Nearest neighbor and hard sphere models in continuum percolation. Random Structures Algorithms 9, 295-315.

[12] Holroyd, A. E. And Peres, Y. (2003). Trees and matchings from point processes. Eletron. Commun. Prob. 8, $17-27$.

[13] Holroyd, A. E. And Soo, T. (2013). Insertion and deletion tolerance of point processes. Electron. J. Prob. 18, $24 \mathrm{pp}$.

[14] Holroyd, A. E., Pemantle, R., Peres, Y. and Schramm, O. (2009). Poisson matching. Ann. Inst. H. Poincaré Prob. Statist. 45, 266-287.

[15] Jonasson, J. (2009). Invariant random graphs with iid degrees in a general geography. Prob. Theory Relat. Fields 143, 643-656.

[16] Kallenberg, O. (2002). Foundations of Modern Probability, 2nd edn. Springer, New York.

[17] Meester, R. AND Roy, R. (1994). Uniqueness of unbounded occupied and vacant components in Boolean models. Ann. Appl. Prob. 4, 933-951. 